\title{
Usage of synthetic tendons in tendon reconstruction
}

\author{
Shalimar Abdullah \\ From 10th Congress of the Asia-Pacific Federation of Societies of Surgery fo the Hand and the 6th Congress \\ of Asia-Pacific Federation of Societies of Hand Therapists \\ Kuala Lumpur, Malaysia. 2-4 October 2014
}

Damaged tendons or loss of tendon poses a major surgical reconstructive problem. The conventional method is harvest donor tendons from palmaris longus, plantaris or tensor fasciae lata. Synthetic tendons has been used in Achilles tendon repair with studies on equine subjects. We present successful use in three patients with extensive tendon loss secondary to polytrauma. Additionally we have conducted an in-vitro study to analyse the ingrowth of tenocytes into the synthetic tendon.

The synthetic tendon utilized in our study is Ortho-tape, a polyethylene terephthalate (polyester) (Neoligaments, Leeds, UK). Its construct is non-absorbable, woven with longitudinal and transverse fibres crossing at right angles. It has an "open structure" acting as a scaffold allowing bone and tissue ingrowth. It is not to be confused with the "permanent type" implant such as Gore-tex which is designed to last a lifetime with no contribution from the host tissue or new tissue growth.

Artificial ligaments clinically available has been carbon (Johnson and Johnson), carbon and polyester (Surgicraft), Leeds-Keio polyester (Neoligaments), Dacron (StrykerMeadox), bovine glutaraldehyde-fixed xenograft (Xenotech) and Gore-Tex polytetrafluoroethylene (WL Gore).

Synthetic textile implants do not require donor harvesting shortening surgical procedural time and allows retention of initial strength whilst autografts undergo a necrotic stage before eventually recovering over a long period of time. Additionally, donor tendon grafts have varying properties and dimensions with limited availability.

Textile implants has been utilized in ACL reconstruction and patellar ligament reconstruction [1-3] however there has been a decline in usage do to some concerns of synovitis after a prolonged duration 4 . Hence some

\footnotetext{
Department of Orthopaedics, Universiti Kebangsaan Malaysia, Kuala Lumpur,
} 56000 Malaysia

\section{Clinical cases}

Our first patient sustained a degloving wound of the dorsum of her left hand with loss of the proximal half of the $1^{\text {st }}$ to $5^{\text {th }}$ metacarpal bones and distal carpal bones. The EDC to index to small fingers were all absent with a tendon gap of $7 \mathrm{~cm}$. After multiple wound debridements, and stabilization with K-wires, she underwent wound coverage with a radial forearm flap from the opposite forearm. Six months later, we proceeded to plate a block of iliac crest bone graft into the empty bony defect. Subsequently after a further six months, we utilized synthetic textile implants to replace the extensor tendon loss (Figure 1).

Initially this was meant to be the first stage of a tendon procedure to be replaced with her own palmaris and plantaris grafts. However her function was good and she declined the second stage. She was able to extend all her fingers fully with a DASH score of 18.8 at years follow-up. Dynamometer grip force was $2 \mathrm{~kg}$. Pinch grip was $2.5 \mathrm{~kg}$.

Our second patient was a 43-year-old lady who sustained an industrial accident with near total amputation of her right wrist with an open fracture Grade IIIC of her right radius and ulna. Her EDC to her index to little fingers were all avulsed. Her DASH score post-operatively was 58.6. Dynamometer was $0 \mathrm{~kg}$ and pinch force was $0 \mathrm{~kg}$. She was able to gain extension at the MCPJ of 30 degrees at 3 years follow-up.

In both patients, the Orthotape was measured to length from the tendinous origin of the muscle till the distal remnant of existing tendon. Appropriate tension was utilized before the Orthotape was sutured using the Pulvertaft weave augmented with Prolene 3/0 sutures at 


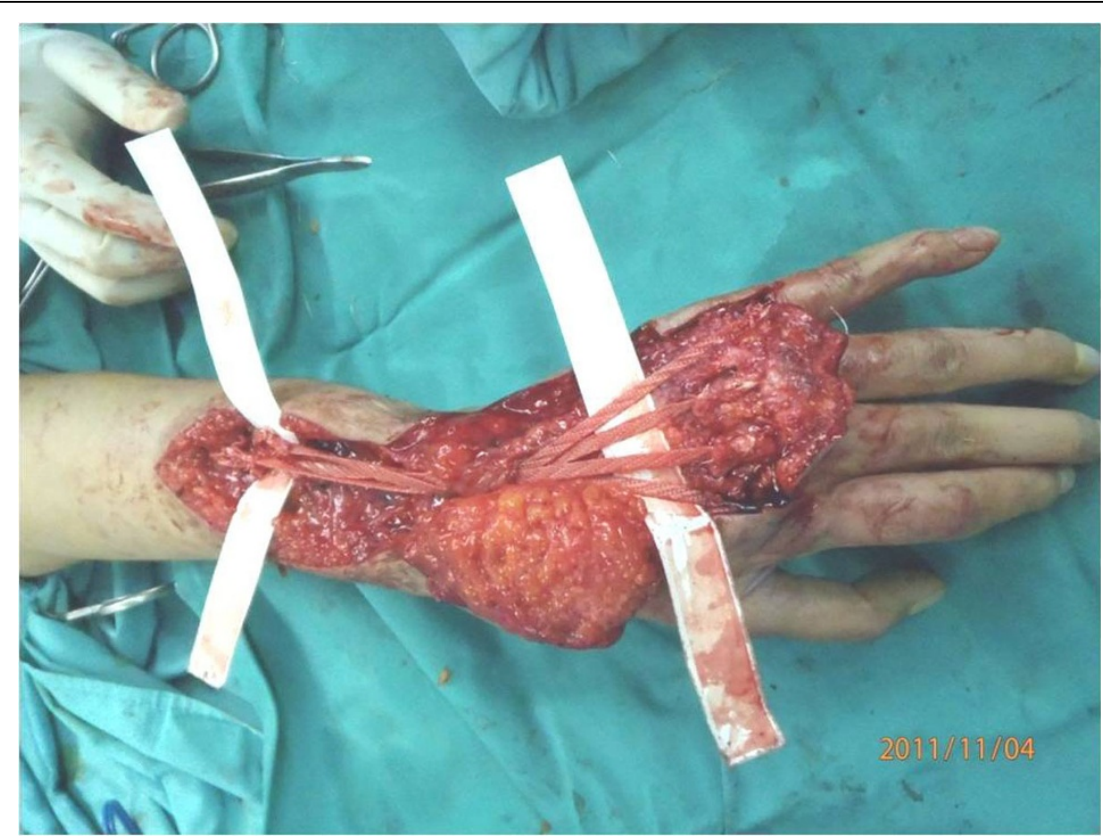

Figure 1 Synthetic textile implant utilized to reconstruct massive extensor tendon loss

both these ends. Any pulleys or retinaculum was reconstructed along the length of the Orthotape. Patients underwent immediate physiotherapy the next day postoperatively.

\section{In-vitro study}

Subsequently we explored seeding a three dimensional tensile scaffold to human tendons of the hand with human tenocyte cells in vitro and incubating it in culture medium for 2,4,6 and 8 weeks. Our methodology consisted of harvesting tendons from patients indicated for upper limb amputation following consent of the patient. Both ends of a $0.5 \mathrm{~cm}$ strip of synthetic tendon will have a $1 \mathrm{~cm}$ strip of flexor or extensor tendon sutured to it. The whole specimen will then be immersed and cultured in growth medium. Eight samples were prepared and maintained in a $\mathrm{CO} 2$ incubator with a change of growth medium thrice a week.

At the end of 2, 4, 6 and 8 weeks, samples of the tendon from the designated weeks were fixed with formalin overnight, embedded in paraffin and cut from paraffin blocks using a microtome. Haemoxylin and eosin staining were performed. Tissue characteristics and percentage of growth of tenocytes were examined.

Results show no tenocyte growth in the first 2 weeks of incubation. However at 4 weeks, tenocytes were seen migrating towards the junction between the tendon and synthetic scaffold. At 6 weeks, more tenocytes were seen growing into the synthetic scaffold and at 8 weeks, tenocytes were seen in abundance in the synthetic tendon.
Thus tenocytes from human tendon are able to gradually proliferate into a synthetic tendon scaffold.

\section{Conclusion}

We recommend usage of synthetic polyester tendon implants as an alternative to tendon autografts in hand reconstruction with massive tendon loss.

Published: 19 May 2015

\section{References}

1. Jennings AG, Sefton GK: Chronic rupture of tendo Achillis. Long-term results of operative management using polyester tape. J Bone JointSurg 2002, 84(3):361-363.

2. Zaffagnini, De Pasquale V, Montanari C, R Strocchi, Marcacci M, et al: Histological and ultrastructural evaluation of Leeds-Keio ligament 20 years after implant: A Case report. Knee Surg Sports Traumatol ArthrosC 1997, 5(2):89-94.

3. Fujikawa K, Iseki F, Seedhom BB: Arthroscopy after anterior cruciate reconstruction with the Leeds-Keio ligament. J Bone Joint Surg Br 1989, 71(4):566-570.

4. Murray AW, Macnicol MF: 10-16 year results of Leeds-Keio anterior cruciate ligament reconstruction. Knee 2004, 11(1):9-14.

doi:10.1186/1753-6561-9-S3-A68

Cite this article as: Abdullah: Usage of synthetic tendons in tendon reconstruction. BMC Proceedings 2015 9(Suppl 3):A68. 